\title{
Feature-level Rating System using Customer Reviews and Review Votes
}

\author{
Koteswar Rao Jerripothula, Member, IEEE, Ankit Rai, Kanu Garg, and Yashvardhan Singh Rautela
}

\begin{abstract}
This work studies how we can obtain featurelevel ratings of the mobile products from the customer reviews and review votes to influence decision making, both for new customers and manufacturers. Such a rating system gives a more comprehensive picture of the product than what a product-level rating system offers. While product-level ratings are too generic, feature-level ratings are particular; we exactly know what is good or bad about the product. There has always been a need to know which features fall short or are doing well according to the customers perception. It keeps both the manufacturer and the customer well-informed in the decisions to make in improving the product and buying, respectively. Different customers are interested in different features. Thus, feature-level ratings can make buying decisions personalized. We analyze the customer reviews collected on an online shopping site (Amazon) about various mobile products and the review votes. Explicitly, we carry out a feature-focused sentiment analysis for this purpose. Eventually, our analysis yields ratings to 108 features for $4 k+$ mobiles sold online. It helps in decision making on how to improve the product (from the manufacturers perspective) and in making the personalized buying decisions (from the buyers perspective) a possibility. Our analysis has applications in recommender systems, consumer research, etc.
\end{abstract}

Index Terms-Recommender systems, natural language processing, sentiment analysis, cellular phones, reviews, decision making, text mining, web mining.

\section{INTRODUCTION}

With the rise of the internet and the kind of busy lifestyles people have today, online shopping has become a norm. Customers often rely on the online ratings of the previous customers to make their decisions. However, most of these ratings on the online websites are product-level ratings and lack specificity. Although products can be compared based on the product-level ratings available, there is always a class of people who prefer buying the items based on particular features. Such people have to generally go through the entire comments section to know previous customers perceptions [1], [2] of the products features in which they are interested. Considering the number of products present for an item (such as mobile), it becomes a tedious job for a customer to arrive at the best product for himself. Moreover, from a manufacturers perspective, such product level ratings hardly specify what is good or bad about the product [3]. So, if feature level ratings are available, it gives more clarity to the seller on how to improve the product. Given all these benefits, our goal is to develop a feature-level rating system.

Koteswar Rao Jerripothula is with the CSE Department, Indraprastha Institute of Information Technology Delhi (IIIT Delhi), India. The remaining authors are with the CSE Department, Graphic Era, India. Contact Author: Koteswar Rao Jerripothula. Email: koteswar@iiitd.ac.in

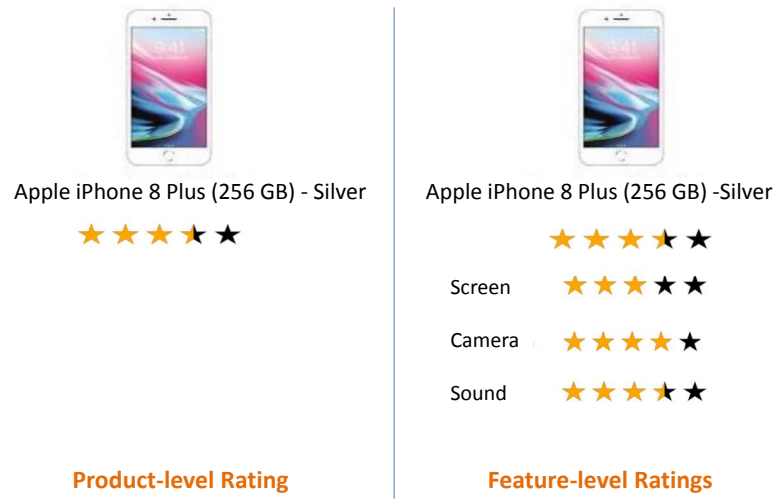

Fig. 1. Product-level Rating System versus Feature-level Rating System: While the first one is very generic, the latter is quite specific.

Although feature-level ratings can also be requested from the customers just like the product-level ratings, it is not a good proposal, for there could be too many features. Instead, it is much more practical to leverage whatever reviews and review votes [4], [5] that are being already given by customers to provide feature-level ratings. The reviews are made up of sentences, and every sentence has some sentiment [6][9] associated with it, viz. positive, neutral, or negative. Also, since they can be separated, we can always extract the sentences describing a particular feature of the product and subsequently obtain sentiment scores over such sentences. By utilizing these sentiment scores as the basis and the review votes as a support, we can build a feature-level rating system that can yield feature-level ratings, as shown in Fig. 1

However, there are few challenges in building such a feature-level rating system. First, we need to determine which features to look for in an item. Another challenge is that there could be many words relating to the same feature; they all need to be clubbed into one feature. Second, we have to pre-process the data as some of the review comments may contain nonEnglish languages, one word, spelling mistakes, etc. Third, we have to devise a way to transform the extracted sentiment scores into an appropriate rating for a feature of a product while incorporating the review votes.

As far as feature identification of an item (such as mobile) is concerned, we go through the word frequency table of the entire customer review data for that item, at least up to a particular frequency. Next, all the related words coming under the same feature are grouped, and the most frequent one is chosen as a representative. We call such representatives as feature keywords. Then, we perform a series of pre-processing 
steps to filter out the unnecessary data, correct the remaining, and turn it into structured data. Each review is broken into sentences, and only relevant sentences are retained. The relevant sentences are passed through sentiment analyzer to generate sentiment scores, which are then adjusted to the ratings. Scores within a particular range are given a specific rating. The ratings of the relevant sentences containing a particular feature are combined using the weighted-average [10] to obtain the final ratings since all opinions are not equally valuable. We leverage review-votes to assign the required weights.

Our contributions are as follows: We develop a featurelevel rating system that takes customer reviews and review votes as inputs and outputs feature-level ratings. We obtain such ratings for as many as $4 \mathrm{k}+$ mobiles sold online in terms of as many as 108 features. We propose votes-aware cumulative rating and votes-aware final rating measures, a new way of accumulating and finalizing the sentiment scores. Although there are no ground truths available, we still manage to evaluate our approach by comparing the final ratings of our phone feature against overall ratings of the phone given by the customers themselves, which leads to remarkable results demonstrating the effectiveness of our method.

\section{RELATED WORK}

Sentiment analysis [11] $-[18]$ has been an active research topic for a long period now. It has applications in health [19][21], politics [22], [23], sports [24], [25], e-commerce [26], [27], etc. In e-commerce, customer reviews can give lots of insights about the products, as shown in [28], [29], through sentiment analysis. Specifically, [11] studies trends of mobile brands on Twitter through sentiment analysis. However, the analysis is restricted to the mobile overall, not to specific features. [30] tries to do so, but for limited products and limited features. While [30] used SVM, a supervised learning algorithm, [31] used ensembling for achieving this. In this paper, we attempt to exploit these customer reviews to provide ratings for as many as 108 features of $4 \mathrm{k}+$ mobile phones sold online while incorporating review votes, which has never been done in the previous studies. Moreover, we do this in an unsupervised way, not supervised or weakly-supervised [32]- 35] way, thanks to the lexical approach of generating sentiment scores for a sentence. Similar to our work, [36] explored digital cameras and television for the same. However, they explore only ten features. [37] explored only Cannon Camera, iPhone 4s, and Mp3 player. However, we explore as many as $4 \mathrm{k}+$ products and provide our recommendations. Nevertheless, to the best of our knowledge, this is the first paper to account for review votes in a feature-level rating system.

\section{Methodology}

The proposed method has four steps: (1) feature selection; (2) pre-processing; (3) relevant sentence extraction; (4) feature-based rating generation. Every step is described in detail in this section.
TABLE I

WE REMOVE ALL OTHER CHARACTERS EXCEPT THESE, FOR THEY HAVE SOME PURPOSE OR OTHER IN DESCRIBING A FEATURE IN A SENTENCE.

\begin{tabular}{|l|l|}
\hline Purpose & Characters \\
\hline Word Formation & A-Za-Z \\
Punctuation &,$::-! ?$ (space) \\
Emoticons & $:-()=* 83><\wedge /[] \#\{\} \mid ; \backslash \&$ \\
\hline
\end{tabular}

\section{A. Feature Selection}

Let us say we collect a dataset of $N$ feature-related words, denoted by $\mathcal{W}=\left\{w_{1}, \cdots, w_{N}\right\}$, by manually going through word frequency table of the entire customer review data on an item (mobile, in our case). In this way, we identify the features in which people are generally interested. Note that we neglect the words having their frequency less than $0.02 \%$ of the total number of reviews in the review data, which means they are rarely discussed feature-related words and can, therefore, be neglected. Let us say the corresponding frequencies of the feature-related words form another set denoted as $\mathcal{Z}=\left\{z_{1}, \cdots, z_{N}\right\}$. Since the feature-related words related to a particular feature should be clubbed into one feature, we define a relationizer function denoted as $\mathcal{R}\left(\mathcal{W}, w_{i}\right)$, which returns a set of all the related words of $w_{i}$ in $\mathcal{W}$, including itself. Note that the relationizer function discussed here as a matter of notation is manual. We now define our feature dataset, denoted by $\mathcal{F}$, as a set of such distinct sets of related feature words, as defined below:

$$
\mathcal{F}=\left\{\mathcal{R}\left(\mathcal{W}, w_{i}\right)\right\}_{i \in\{1, \cdots, N\}}
$$

where we iterate through all the words in $\mathcal{W}$ and form distinct sets of the related words using the relationizer function. Since different related words will form the same sets, the duplicates will be removed to make the sets left distinct. Now, let $\mathcal{F}_{k}$ be the $k^{\text {th }}$ feature words set in the $\mathcal{F}$ feature dataset. In any $\mathcal{F}_{k}$, a representative feature word is selected to identify the whole feature words set. Let us call such representatives as feature keywords. The most frequent feature word in the set is chosen as the representative (inspired by [38]) or feature keyword to assign its name to the set, as shown below:

$$
\mathcal{F}_{k} \leftarrow w_{i} \mid i=\max \left(\left\{\mathcal{Z}(i) \mid w_{i} \in \mathcal{F}_{k}\right\}\right)
$$

where the feature set is assigned a name with the word that has the maximum frequency in the set. From now on, abusing notations a bit, $\mathcal{F}_{k}$ can mean both the $k^{\text {th }}$ feature set (a set of related feature words) and its keyword (or set's name), according to the context.

\section{B. Pre-processing}

The review comments data is generally unstructured, for it is written by the customers online. Our goal now is to convert this unstructured data into structured data in our pre-processing steps, which means useful data is extracted, disintegrated, and corrected.

Note that the data retained after removing the characters that are useless for our purpose is what we mean as useful data. While inspecting the reviews for figuring out the features to 


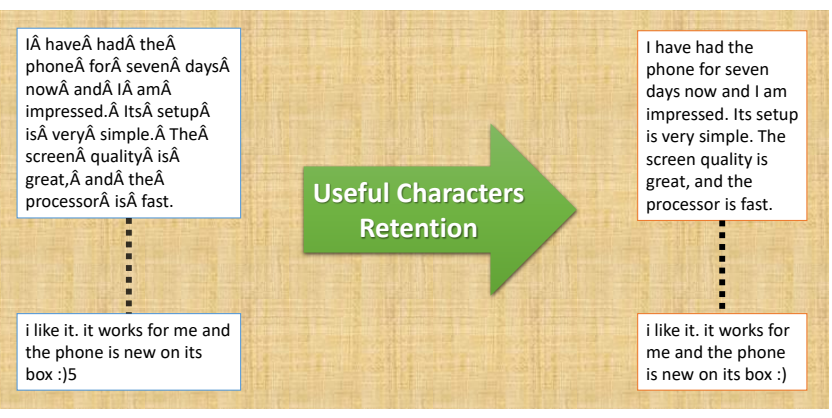

Fig. 2. Pre-processing: Useful Characters Retention, where unnecessary characters are removed in this illustration.

work with, we also noticed how people praise or criticize. People often use the characters required for adjective words, punctuation, or emoticons. While we retain the characters required for word formation, punctuation, and emoticons, we remove all other characters, including numbers, as shown in Fig. 2. In Table. If we give the information regarding what all characters are retained. After that, we remove any entries which are left empty because we have no use of them in the feature-level rating system. Let us consider that, for a product (not an item), we denote product review data as $\mathcal{D}=\left\{C_{1}, C_{2}, \cdots, C_{m}\right\}$, comprising of $m$ useful review comments. Note that when we said customer review data of the item in the last section for feature selection, we meant review data of all the mobiles. In contrast, $\mathcal{D}$ is the review data of just the mobile product under consideration.

By corrected data, we mean the data obtained after correcting the related feature words issue and spelling correction in the useful data just extracted. To correct the data in such a manner, we need to disintegrate the reviews into words and process them separately. We use the NLTK package of python for this purpose. It helps in disintegrating the reviews into words as tokens while neglecting the spaces. It considers even period (.) as a token, which becomes useful later while breaking the comments into sentences. Each comment can now be represented as a set of tokens, i.e. $C_{j}=\left\{t_{j}^{1}, t_{j}^{2}, \cdots, t_{j}^{\left|C_{j}\right|}\right\}$, where $\left|C_{j}\right|$ denotes the number of tokens obtained in $C_{j}$ and $t_{j}^{i}$ represents $i^{t h}$ token of $j^{t h}$ comment. We correct any token $t_{j}^{i}$ of the useful data in the following manner:

$$
t_{j}^{i}= \begin{cases}\mathcal{F}_{k}, & \text { if } S\left(t_{j}^{i}\right) \in \mathcal{F}_{k} \text { or } t_{i} \in \mathcal{F}_{k}, \forall \mathcal{F}_{k} \in \mathcal{F} \\ S\left(t_{j}^{i}\right), & \text { otherwise }\end{cases}
$$

where $S(\cdot)$ represents spelling correcting function (using autocorrect package of python). If a token before or after spelling correction matches with any of the members in any of our feature words sets, we replace it with the feature keyword of that set; otherwise, we replace it with the corrected token. In this way, we take care of both the related words issue (by replacing with keywords) and the spelling correction issue simultaneously. The illustrations of spelling and keywords correction are given in Fig. 3 and Fig. 4 Thus, with the useful data extracted, disintegrated, and corrected, our reviews data for a product becomes structured. Now, we can say that $t_{j}^{i}$ is $i^{\text {th }}$ token of $j^{\text {th }}$ comment of $\mathcal{D}$.

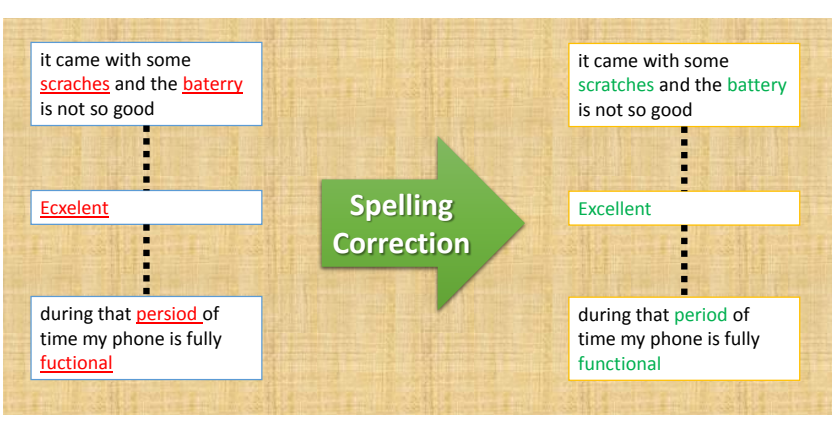

Fig. 3. Pre-processing: Spelling Correction, where misspelled words like 'scraches', 'baterry', 'Ecxelent', 'persiod' and 'fuctional' are corrected in this illustration.

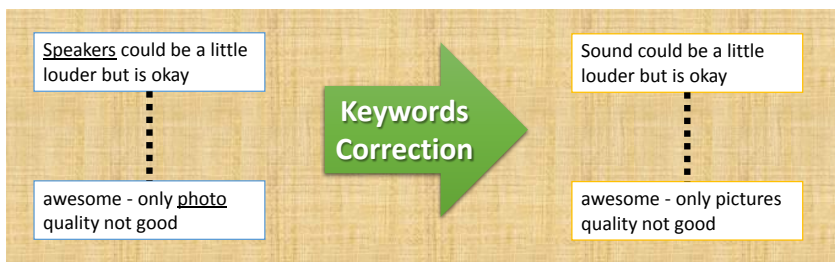

Fig. 4. Pre-processing: Keyword Correction, where feature words like 'speakers' and 'photo' are corrected to their respective feature keywords, 'sound' and 'pictures' in this illustration.

\section{Relevant Sentence Extraction}

Since customer review data is now structured, we can group the continuous tokens present in a review as a sentence, as shown below in the definition of a set of sentences $X_{j}$ derived from $C_{j}$ :

$$
\begin{array}{r}
X_{j}=\left\{\left(t_{j}^{u}, \cdots, t_{j}^{v}\right) \mid\left(t_{j}^{v}, t_{j}^{u-1}\right)=\because,\left(t_{j}^{u}, \cdots, t_{j}^{v-1}\right) \neq{ }^{\prime},\right. \\
\left.(u, v) \in\left\{1, \cdots,\left|C_{j}\right|\right\} \text { and } v>u\right\}
\end{array}
$$

where we call a group of continuous tokens as a sentence if the last and previous-to-beginning token are periods (.), and if all other tokens in that group are not periods (.). However, not all the sentences are relevant for feature-based rating. We define if a sentence $X_{j}^{l}, l^{t h}$ sentence in $X_{j}$, is relevant or not in the following way:

$$
\rho\left(X_{j}^{l}\right)=\left\{\begin{array}{l}
1, \text { if } \mathcal{F}_{k} \in X_{j}^{l} \text { for any } \mathcal{F}_{k} \in \mathcal{F} \\
0, \text { otherwise }
\end{array}\right.
$$

where $\rho(\cdot)$ is relevance function for a sentence which outputs 1 if any of our feature keywords are present in the sentence.

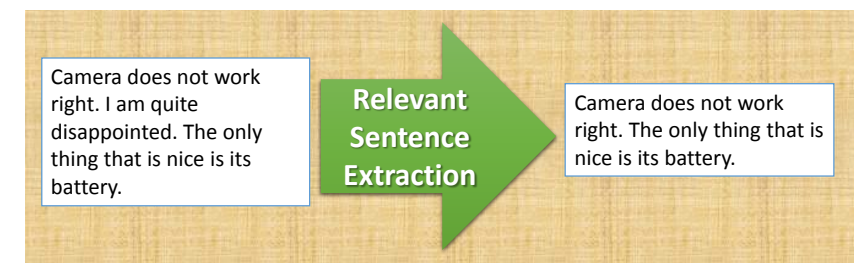

Fig. 5. Relevant Sentence Extraction: Only sentences containing feature keywords are retained. 


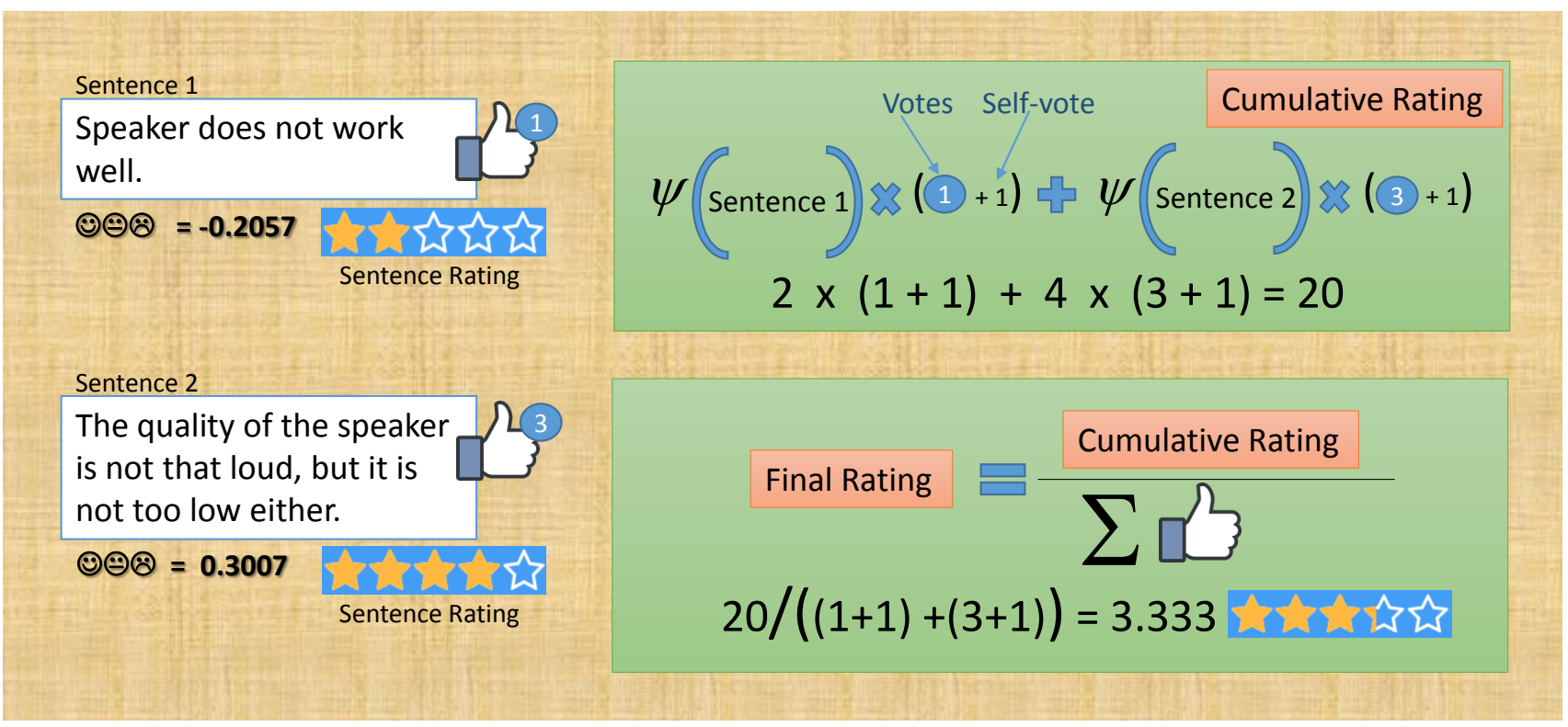

Fig. 6. First, to compute cumulative rating for a feature, we accumulate sentence rating of all the sentences having the feature word (speaker in this case) along with their total votes (other + self). Second, to compute the final rating, we divide the cumulative rating by the total number of votes.

In this way, we extract only relevant sentences. See Fig. 5 for an example.

\section{Feature-based Ratings Generation}

Having extracted relevant sentences, we can go through each sentence to figure out if it mentions a particular feature, say $F_{k}$. If yes, we can extract the emotion of the sentence to score it. For this purpose, we extract sentiment analysis scores [39] for each of these sentences. We use [39] because it accommodates emoticons also while performing the analysis. We use their compound score as the required sentiment analysis score. It ranges between -1 and 1 . We divide this range into five equal parts and assign the ratings progressively, as shown in the Table II. Let the function that computes the sentiment score and assigns the appropriate rating be $\psi(\cdot)$. Then, we compute cumulative rating $(\mathcal{Q}(\cdot))$ for each feature over the entire product review data, i.e., $\mathcal{D}$, in the following manner:

$$
\mathcal{Q}\left(\mathcal{F}_{k}\right)=\sum_{C_{j} \in \mathcal{D}} \sum_{X_{j}^{l} \in X_{j}}^{\rho\left(X_{j}^{l}\right)=1} \psi\left(X_{j}^{l}\right) \times \delta\left(\mathcal{F}_{k} \in X_{j}^{l}\right) \times\left(\phi\left(C_{j}\right)+1\right)
$$

where $\delta(\cdot)$ denotes logical function to check if a sentence consists of the concerned feature or not. During our accumulation, we also consider the number of votes received to review to which the sentence belongs. These votes inform us about the strength of the opinion associated with the reviews. Let $\phi\left(C_{j}\right)$ denote the number of votes received for $C_{j}$. Here, we are assuming that any sentence equally contributes to the strength of the opinion. We adjust the votes by adding 1 to account for self-votes of the customers who originally wrote the reviews. This accumulation is illustrated in Fig. 6. Then,
TABLE II

CONVERSION OF A SENTENCE'S SENTIMENT SCORE TO SENTENCE'S RATING

\begin{tabular}{|c|l|l|}
\hline Sentiment Score & Rating & Meaning \\
\hline-1.0 to -0.6 & 1-star & Terrible \\
\hline-0.6 to -0.2 & 2-star & Poor \\
\hline-0.2 to 0.2 & 3-star & Average \\
\hline 0.2 to 0.6 & 4-star & Very Good \\
\hline 0.6 to 1.0 & 5-star & Excellent \\
\hline
\end{tabular}

we compute our final rating $(\mathcal{A}(\cdot))$ for a feature $\mathcal{F}_{k}$ using the below equation:

$$
\mathcal{A}\left(\mathcal{F}_{k}\right)=\frac{\mathcal{Q}\left(\mathcal{F}_{k}\right)}{\sum_{C_{j} \in \mathcal{D}} \sum_{X_{j}^{l} \in X_{j}}^{\rho\left(X_{j}^{l}\right)=1} \delta\left(\mathcal{F}_{k} \in X_{j}^{l}\right) \times\left(\phi\left(C_{j}\right)+1\right)}
$$

where we divide the cumulative number of stars by the total number of votes received during the accumulation. In this way, we are essentially computing the weighted average [40], where weights are determined by the votes received. So, we now have a feature-level rating for the feature $\mathcal{F}_{k}$ of a product using the customer reviews and review votes. The same proposed methodology can be applied to any number of features, any number of products, and any number of items.

\section{EXPERIMENTS}

In this section, we give details of experiments conducted using the proposed methodology. First, we discuss the dataset used. Then, we discuss the features chosen from the word frequency table of the dataset. At last, we discuss our analysis of the feature-level ratings obtained using the proposed method. 
TABLE III

OUR FEATURES DATASET: THE RELATED WORDS ARE SEPARATED BY || AND LED BY A KEYWORD

|| phone, product, phones, device, cell, item, smartphone, mobile, model, cellphone, products, devices, piece, cellular, smartphones, items, telephone, handset,cellphones || screen, display, glass, screens, lcd, displays, displaying || battery, batteries $\|$ camera, resolution, cameras, pixels, pixel, cam, megapixel, megapixels $\|$ price, money, buying, pay, cost, paid, sold, purchasing, spent, budget, bucks, rate, spending, pricing, pays $\|$ sim, card, dual, sims || apps, app, program, application, widgets, processes, module \|| android, version, operating, ios, versions, software, windows $\|$ case, box, packaged, packing, boxes, packs $\|$ charge, charged, charging, charges, discharges, discharge, charging $\|$ charger, plug, adapter, chargers, plugs || service, services || watch, clock || size, sizes || call, talk, voice, called, talking, dial, speak, outgoing, communications, communicating || wifi | brand || memory, data, space || pictures, picture, photos, pics, photo, image, images, photography || touch, touchscreen $\|$ text, texts, editing, txt, texted || sound, speaker, speakers, sounds, loudspeaker || sd, micro, microsd || network, networks || button, keyboard, buttons, key, keys, typing, qwerty, keypad, keyboards, dials $\|$ music, audio, listen, listening $\|$ internet, online, web, browsing $\|$ light, flash, flashlight, torch || warranty || bluetooth, wireless || video, videos, streaming, stream, fps || settings, setting, setup, configure, configuration, calibration || color, colors, colour || design, build, shape, compact || download, downloaded, downloads $\|$ usb, microusb $\|$ email, emails $\|$ speed, speeds, speedy || headphones, headset, earphones, earphone, headsets || gps || games, gaming || ram || messages, messaging, sms, messenger, msg || cable, cord, connector, cables || manual, instructions, instruction, booklet $\|$ processor, cpu, processors $\|$ specs, specifications, spec $\|$ hardware || fingerprint, finger, fingers, fingerprints || switch, switched, switches || accessories, accessory || weight, bulky, lightweight || sensor, sensors $\|$ face, faces || protection, firmware, protectors, virus, antivirus $\|$ notification, notifier, notifications, prompts || brightness $\|$ jack $\|$ chip, chipset || tested, checked, tests || scanner, qr || files || microphone || navigation, navigating || waterproof || calendar || mms || alarm, alarms || hotspot || graphics, gpu, graphic $\|$ icons, icon $\|$ selfie, selfies $\|$ recharge, recharging || electronics || vibrate, vibration, vibrates, vibrating, shake || recording, recorder || zoom, lens || chat, chatting || ringtone, ringing || heats, overheats, temperature, overheated, temp || voicemail, inbox $\|$ stereo || scroll, slider, slides, swipe $\|$ guarantee, guaranteed $\|$ languages $\|$ multimedia || compatibility $\|$ driver, drivers || pedometer || trackpad || calculator || handsfree || autofocus || otg || troubleshooting, troubleshoot || airplane || mute || syncs || multitask || backlight | permissions || reminders || echo || trackball || panorama || speech || lockscreen || vga ||

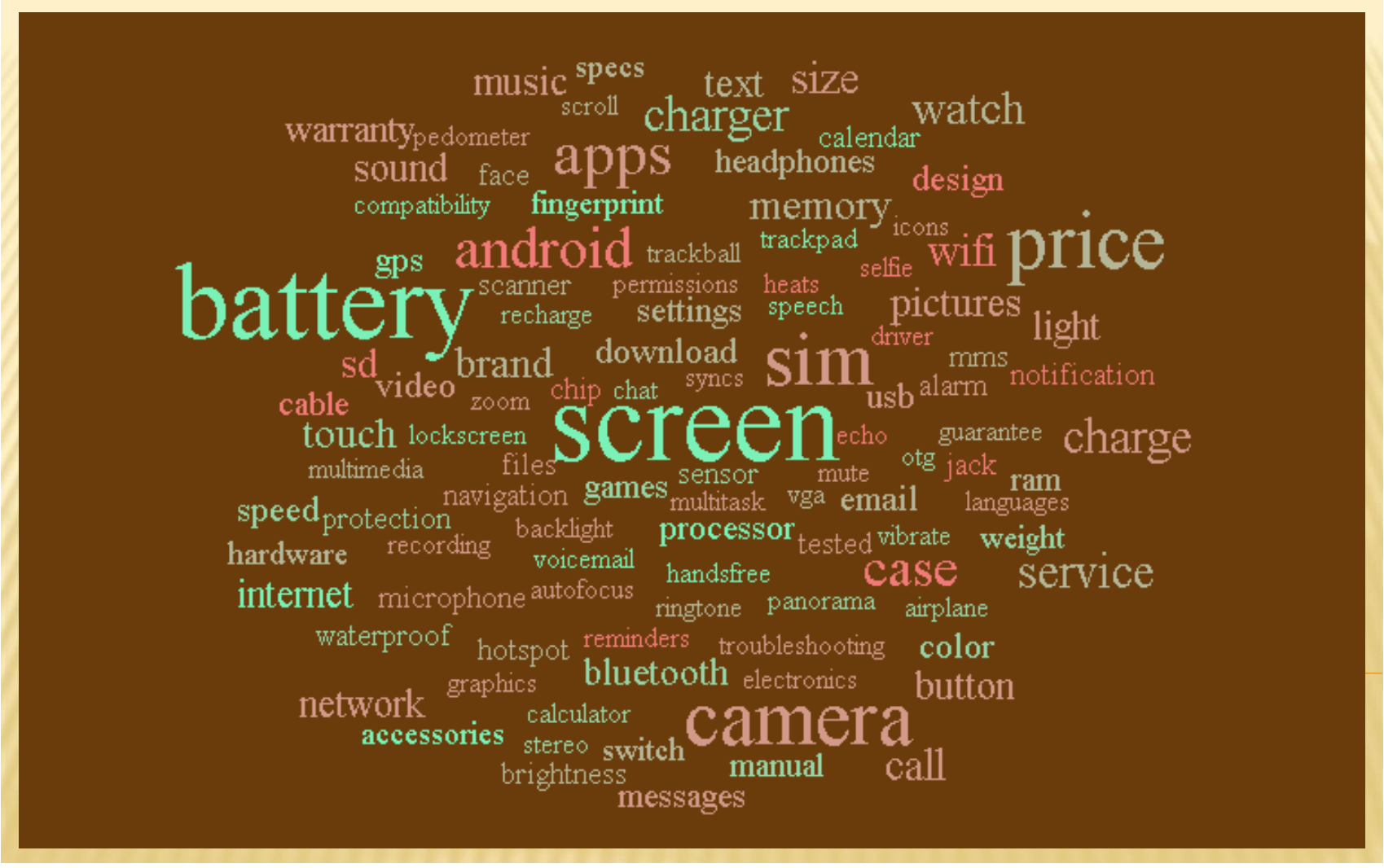

Fig. 7. Word Cloud for our features (except 'phone' feature). 
TABLE IV

OUR SAMPLE RESULTS FOR A PHONE NAMED Nokia C6.

\begin{tabular}{|l|l||l|l|}
\hline Feature & Rating & Feature & Rating \\
\hline \hline alarm & 1.818 & email & 3.107 \\
speed & 4.977 & brightness & 3 \\
phone & 3.524 & button & 3.757 \\
chip & 3.349 & weight & 3.652 \\
music & 3.916 & sensor & 3 \\
camera & 4.032 & face & 4.538 \\
electronics & 1 & service & 3.123 \\
calendar & 3.305 & files & 3.022 \\
navigation & 4.111 & call & 3.785 \\
sim & 3.255 & troubleshooting & 5 \\
multimedia & 3 & cable & 3.848 \\
tested & 3.5 & zoom & 4.788 \\
vibrate & 2 & multitask & 5 \\
network & 3.464 & settings & 3.169 \\
sound & 3.512 & gps & 4.879 \\
internet & 3.985 & hardware & 2.2 \\
charge & 3.216 & touch & 3.66 \\
languages & 3 & stereo & 3 \\
case & 3.454 & echo & 3.714 \\
jack & 3 & headphones & 3.071 \\
price & 3.13 & memory & 3.227 \\
calculator & 2 & hotspot & 3 \\
text & 2.426 & wifi & 4.702 \\
heats & 4 & scroll & 3.705 \\
light & 4.075 & specs & 5 \\
brand & 3.15 & usb & 3.286 \\
icons & 4.667 & manual & 3.614 \\
size & 4.776 & warranty & 2.773 \\
switch & 3.957 & messages & 3.61 \\
apps & 3.738 & download & 3.787 \\
protection & 3.769 & battery & 3.754 \\
android & 3.514 & ringtone & 3.333 \\
chat & 3.917 & pictures & 3.836 \\
watch & 2 & bluetooth & 3.993 \\
fingerprint & 4.206 & accessories & 2 \\
design & 4.6 & games & 4.016 \\
sd & 2.739 & screen & 3.516 \\
mms & 5 & video & 3.24 \\
charger & 3.298 & color & 3.647 \\
\hline & & & \\
\hline
\end{tabular}

\section{A. Dataset}

We apply the proposed method on a dataset named Amazon Reviews: Unlocked Mobile Phones 1 , a dataset extracted by PromptCloud from the Amazon website. It consists of reviews, product-level ratings, and review votes for a total of 4418 mobile phones. There are a total of 413841 reviews present in the dataset, along with the votes obtained by them. So, there are enough reviews to carry out our sentiment analysis and obtain general insights.

\section{B. Features}

In Table III] we list all the words which we select as feature words. As discussed earlier, they have been extracted while observing the word frequency table of the entire dataset. The feature words which are related are separated from others using $\|$. Many of the words are just plurals of the already existing words. The related feature words are led by a feature keyword (represented in blue color), which is most prominent in the dataset amongst all the related feature words. Note that

\footnotetext{
${ }^{1}$ https://www.kaggle.com/PromptCloudHQ/amazon-reviews-unlockedmobile-phones/data
}

TABLE V

TOP FEW PHONES AFTER RANKING THE PHONES ACCORDING TO THE NUMBER OF FEATURES IN WHICH THEY ARE THE BEST, AS PER OUR RATINGS.

\begin{tabular}{|l|l|}
\hline Phone & $\begin{array}{l}\text { No. of } \\
\text { features }\end{array}$ \\
\hline \hline Nokia N9 - Black & 17 \\
\hline ASUS ZenFone 3 ZE552KL (SHIMMER GOLD) & 15 \\
\hline Asus ZenFone 3 ZE552KL Moonlight White & 14 \\
\hline 5.5” JUNING Blue & 14 \\
\hline JUNING 7-Inch - Black & 14 \\
\hline 5.5” JUNING Black & 14 \\
\hline 5.5” JUNING White & 14 \\
\hline Asus ZenFone 3 ZE552KL Sapphire Black & 14 \\
\hline LG V10 H962 64GB Ocean Blue, 5.7” & 12 \\
\hline SKY Devices Platinum Series 5.0W - Silver & 12 \\
\hline LG V10 H962 64GB Black, 5.7” & 12 \\
\hline Sony Ericsson W995a Walkman (Progressive Black) & 12 \\
\hline BLU Life View L110X 5.7-Inch (Blue) & 12 \\
\hline THL 5000 5" FHD IPS MTK6592T (Black) & 12 \\
\hline 5.5” MTK6580 JUNING GSM/3G Black & 12 \\
\hline LG V10 H962 64GB 5.7-Inch (Brown Beige) & 12 \\
\hline SKY Devices Platinum Series 5.0W - White & 12 \\
\hline LG V10 H962 64GB 5.7-Inch (Opal Blue) (Blue White) & 12 \\
\hline LG V10 H962 64GB White, 5.7” & 12 \\
\hline Huawei P9 Lite VNS-L22 5.2-Inch (BLACK) & 11 \\
\hline LG Electronics G3 Stylus D690 (Black Titanium) & 11 \\
\hline Huawei Mate 8 32GB 6-Inch (Silver) & 11 \\
\hline Huawei Mate 8 NXT-L29 32GB 6-Inch (Space Gray) & 11 \\
\hline LG VX8500 Chocolate Phone (Verizon Wireless) & 11 \\
\hline ASUS Zenfone 6 A600CG 6-inches White & 11 \\
\hline Futuretech A6 4.5 Inch Mtk6582 (Yellow) & 11 \\
\hline Nokia C7 Unlocked Quadband Smartphone & 11 \\
\hline Huawei P9 Lite VNS-L22 5.2-Inch (WHITE) & 10 \\
\hline ASUS ZENFONE 6 A601CG 6" (Black) & 10 \\
\hline Samsung Galaxy S2 PLUS i9150P blue-grey & 10 \\
\hline Futuretech A6 4.5 Inch Mtk6582 (black) & 11 \\
\hline Nokia Asha 302 & 11 \\
\hline ZTE Axon Pro Phthalo Blue & 11 \\
\hline Smart watch, GEEKERA Watch Phone( Black ) & 10 \\
\hline Yezz Andy 5E - (White ) & 10 \\
\hline Nokia Lumia 1520 - Black & 10 \\
\hline Nokia N82 (Silver) & 10 \\
\hline Huawei P9 Lite VNS-L22 (GOLD) & 10 \\
\hline Blackberry Torch 9800 - Black & 10 \\
\hline HTC One Mini 2 16GB - Silver & 10 \\
\hline ZTE Spro 2 Smart Projector (Silver) & 10 \\
\hline Nokia N79 (Silver) & 10 \\
\hline Cubot X15 5.5” Inches & 10 \\
\hline Samsung Evergreen A667 - Black & 10 \\
\hline Straight Talk Phone X2 & 10 \\
\hline ZTE Axon Pro & 10 \\
\hline Nokia Lumia 1520 - Red & 10 \\
\hline Honor 8 Dual Camera - Pearl White & 10 \\
\hline ZTE Axon Pro, A1P133, 32 GB, Chromium Silver \\
\hline ZTE Axon Pro, 64 GB, Chromium Silver & 10 \\
\hline Unnecto Air 5.5 (Gray) & 10 \\
\hline LG G3 Stylus 3G D690 (White) & 10 \\
\hline Nokia X3 & 10 \\
\hline OnePlus White 5.5 inch & 10 \\
\hline
\end{tabular}

some feature words like sd, vga, otg, etc., are not common and likely to be misunderstood as incorrect words by autocorrector. As a result, when autocorrection is applied, they may get changed to some so-called correct words. To avoid this from happening, in Eqn.(3), we check the original token's presence as well in our feature words dataset, not just the corrected token.

Note that we consider the entire phone as one of the features 
TABLE VI

NUMBER OF PHONES WITH DIFFERENT INTEGER RATINGS FOR EACH FEATURE

\begin{tabular}{|c|c|c|c|c|c|c|c|c|c|c|c|}
\hline Feature & 1-star & 2-star & 3-star & 4-star & 5-star & Feature & 1-star & 2-star & 3-star & 4-star & 5-star \\
\hline phone & 12 & 88 & 1897 & 1925 & 219 & protection & 30 & 133 & 416 & 285 & 108 \\
\hline screen & 28 & 213 & 1161 & 1162 & 333 & notification & 21 & 100 & 214 & 143 & 43 \\
\hline battery & 57 & 305 & 1346 & 813 & 267 & brightness & 5 & 8 & 82 & 199 & 245 \\
\hline camera & 38 & 219 & 706 & 1049 & 466 & jack & 23 & 113 & 247 & 116 & 50 \\
\hline price & 39 & 198 & 1101 & 1575 & 378 & chip & 17 & 105 & 287 & 165 & 71 \\
\hline $\operatorname{sim}$ & 36 & 260 & 1336 & 811 & 182 & tested & 18 & 132 & 515 & 328 & 134 \\
\hline apps & 35 & 203 & 1104 & 797 & 216 & scanner & 5 & 25 & 113 & 61 & 54 \\
\hline android & 32 & 237 & 1097 & 803 & 219 & files & 8 & 69 & 211 & 143 & 104 \\
\hline case & 30 & 242 & 1128 & 853 & 242 & microphone & 34 & 160 & 209 & 83 & 54 \\
\hline charge & 52 & 319 & 1469 & 489 & 128 & navigation & 15 & 65 & 198 & 154 & 112 \\
\hline charger & 63 & 324 & 1260 & 458 & 119 & waterproof & 8 & 35 & 125 & 97 & 48 \\
\hline service & 51 & 227 & 848 & 725 & 331 & calendar & 11 & 34 & 172 & 100 & 61 \\
\hline watch & 22 & 98 & 311 & 282 & 171 & $\mathrm{mms}$ & 4 & 27 & 55 & 51 & 21 \\
\hline size & 10 & 52 & 384 & 802 & 526 & alarm & 48 & 248 & 122 & 39 & 27 \\
\hline call & 39 & 244 & 1194 & 730 & 197 & hotspot & 12 & 20 & 144 & 83 & 41 \\
\hline wifi & 36 & 183 & 573 & 320 & 137 & graphics & 10 & 21 & 88 & 126 & 197 \\
\hline brand & 29 & 115 & 705 & 598 & 314 & icons & 16 & 116 & 251 & 163 & 52 \\
\hline memory & 42 & 240 & 1124 & 639 & 214 & selfie & 11 & 26 & 90 & 135 & 125 \\
\hline pictures & 28 & 202 & 775 & 948 & 394 & recharge & 24 & 77 & 266 & 112 & 33 \\
\hline touch & 55 & 235 & 614 & 529 & 209 & electronics & 16 & 51 & 98 & 111 & 62 \\
\hline text & 39 & 204 & 842 & 468 & 192 & vibrate & 18 & 115 & 287 & 118 & 29 \\
\hline sound & 62 & 300 & 585 & 787 & 322 & recording & 28 & 33 & 141 & 122 & 85 \\
\hline sd & 25 & 119 & 525 & 384 & 160 & zoom & 19 & 81 & 163 & 130 & 78 \\
\hline network & 40 & 185 & 845 & 484 & 141 & chat & 15 & 24 & 107 & 133 & 82 \\
\hline button & 43 & 269 & 1109 & 595 & 148 & ringtone & 16 & 60 & 161 & 81 & 57 \\
\hline music & 23 & 155 & 573 & 574 & 270 & heats & 25 & 136 & 225 & 104 & 53 \\
\hline internet & 39 & 213 & 943 & 579 & 207 & voicemail & 5 & 68 & 133 & 53 & 37 \\
\hline light & 36 & 117 & 607 & 630 & 317 & stereo & 4 & 24 & 67 & 66 & 55 \\
\hline warranty & 47 & 277 & 701 & 221 & 48 & scroll & 22 & 91 & 253 & 170 & 93 \\
\hline bluetooth & 18 & 146 & 485 & 349 & 138 & guarantee & 13 & 33 & 86 & 212 & 78 \\
\hline video & 18 & 139 & 514 & 620 & 318 & languages & 5 & 58 & 104 & 62 & 31 \\
\hline settings & 15 & 195 & 749 & 554 & 168 & multimedia & 1 & 22 & 49 & 43 & 42 \\
\hline color & 32 & 104 & 305 & 565 & 509 & compatibility & 11 & 43 & 89 & 81 & 37 \\
\hline design & 13 & 81 & 282 & 813 & 632 & driver & 13 & 26 & 124 & 74 & 31 \\
\hline download & 22 & 155 & 635 & 457 & 165 & pedometer & 0 & 0 & 25 & 17 & 4 \\
\hline usb & 26 & 95 & 251 & 114 & 37 & trackpad & 4 & 10 & 13 & 7 & 3 \\
\hline email & 39 & 140 & 654 & 359 & 145 & calculator & 2 & 24 & 57 & 42 & 32 \\
\hline speed & 27 & 90 & 412 & 458 & 344 & handsfree & 7 & 18 & 52 & 60 & 18 \\
\hline headphones & 62 & 255 & 629 & 393 & 144 & autofocus & 8 & 36 & 38 & 30 & 28 \\
\hline gps & 29 & 31 & 104 & 74 & 46 & otg & 0 & 6 & 4 & 6 & 1 \\
\hline games & 22 & 89 & 330 & 434 & 256 & troubleshooting & 11 & 30 & 101 & 50 & 14 \\
\hline ram & 7 & 27 & 220 & 151 & 88 & airplane & 2 & 23 & 59 & 37 & 6 \\
\hline messages & 27 & 198 & 688 & 299 & 107 & mute & 6 & 17 & 68 & 28 & 17 \\
\hline cable & 53 & 247 & 635 & 321 & 84 & syncs & 3 & 21 & 25 & 32 & 19 \\
\hline manual & 38 & 267 & 656 & 299 & 122 & multitask & 0 & 12 & 37 & 18 & 45 \\
\hline processor & 22 & 73 & 333 & 344 & 180 & backlight & 6 & 13 & 35 & 19 & 17 \\
\hline specs & 17 & 69 & 344 & 342 & 231 & permissions & 0 & 5 & 49 & 10 & 11 \\
\hline hardware & 23 & 160 & 289 & 221 & 163 & reminders & 0 & 18 & 29 & 21 & 10 \\
\hline fingerprint & 35 & 134 & 437 & 320 & 138 & echo & 14 & 22 & 54 & 15 & 4 \\
\hline switch & 45 & 152 & 610 & 346 & 147 & trackball & 1 & 3 & 9 & 15 & 0 \\
\hline accessories & 17 & 92 & 410 & 330 & 186 & panorama & 0 & 1 & 25 & 20 & 23 \\
\hline weight & 15 & 61 & 355 & 365 & 327 & speech & 4 & 7 & 22 & 24 & 15 \\
\hline sensor & 20 & 71 & 211 & 171 & 77 & lockscreen & 1 & 5 & 7 & 11 & 13 \\
\hline face & 23 & 102 & 206 & 181 & 148 & vga & 0 & 0 & 5 & 2 & 7 \\
\hline
\end{tabular}

as well, and this is the most discussed feature, as expected. Such consideration helps us evaluate the performance of the proposed method by comparing the feature level ratings of our phone feature with the corresponding product-level ratings already available in the dataset. We also give word cloud for actual feature keywords (i.e., except phone) in Fig. 7 . Larger the keyword, most discussed it is in the reviews. It is clear from the word cloud that battery, screen, price, camera, sim, and apps are some of the most discussed features in phones.

\section{Ratings}

We give sample feature-level ratings of our proposed method in Table IV] it is for Nokia C6. It can be noted how the proposed method can rate a phone on a vast number of features, and this can certainly help consumers [41] in making their decisions on buying their mobile phones. Since we can get feature-level ratings for all the phones, to summarize our results, we report the number of phones close to different integer ratings (e.g., 2-star) for each feature in Table VI We obtain this by rounding off the ratings calculated. It is clear from the Table that most of the phones get 3-star ratings, 
TABLE VII

THE BEST PHONES WE RECOMMEND FOR DIFFERENT FEATURES.

\begin{tabular}{|c|c|c|c|}
\hline Features & Best Phones & Features & Best Phones \\
\hline phone & ASUS ZenFone 3 ZE552KL 5.5-inch (SHIMMER GOLD) & protection & SKY Devices Platinum Series 5.0W - Silver \\
\hline screen & LG VX8500 Chocolate Phone (Verizon Wireless) & notification & Blackberry Z10 16GB - Black \\
\hline battery & JUNING 7-Inch - Black & brightness & ASUS Zenfone 6 A600CG White \\
\hline camera & Huawei P9 Lite VNS-L22 5.2-Inch (BLACK) & jack & Nokia N9 16 GB MeeGo OS - Black \\
\hline price & THL 5000 5” FHD IPS MTK6592T (Black) & chip & Nokia N9 16 GB MeeGo OS - Black \\
\hline $\operatorname{sim}$ & Huawei P9 Lite VNS-L22 5.2-Inch (BLACK) & tested & Nokia N9 16 GB MeeGo OS - Black \\
\hline apps & ASUS ZenFone 3 ZE552KL 5.5-inch (SHIMMER GOLD) & scanner & ASUS ZenFone 3 ZE552KL 5.5-inch (SHIMMER GOLD) \\
\hline android & LG V10 H962 64GB Ocean Blue, Dual Sim, 5.7” & files & ASUS ZenFone 3 ZE552KL 5.5-inch (SHIMMER GOLD) \\
\hline case & Nokia N9 16 GB MeeGo OS - Black & microphone & 5.5" JUNING Blue \\
\hline charge & Nokia N9 16 GB MeeGo OS - Black & navigation & Nokia N9 16 GB MeeGo OS - Black \\
\hline charger & $5.5 \%$ JUNING Blue & waterproof & Sony Xperia Z1 (C6902) - Black \\
\hline service & THL 5000 5" FHD IPS MTK6592T (Black) & calendar & 5.5" JUNING Blue \\
\hline watch & $5.5 "$ JUNING Blue & mms & New Genuine Nokia X3-00 Unlocked GSM X3 \\
\hline size & $5.5 ”$ JUNING Blue & alarm & BLU Life View L110X 5.7-Inch (Blue) \\
\hline call & ASUS ZenFone 3 ZE552KL 5.5-inch (SHIMMER GOLD) & hotspot & 5.5” JUNING Blue \\
\hline wifi & THL 5000 5" FHD IPS MTK6592T (Black) & graphics & JUNING 7-Inch - Black \\
\hline brand & JUNING 7-Inch - Black & icons & Sony Ericsson W995a Walkman (Progressive Black) \\
\hline memory & ASUS ZenFone 3 ZE552KL 5.5-inch (SHIMMER GOLD) & selfie & 5.5" JUNING Blue \\
\hline pictures & ASUS ZenFone 3 ZE552KL 5.5-inch (SHIMMER GOLD) & recharge & BLU Life View L110X 5.7-Inch (Blue) \\
\hline touch & $5.5 \%$ JUNING Blue & electronics & Samsung S7 Galaxy (SM-G930UZKAXAA) \\
\hline text & ASUS ZenFone 3 ZE552KL 5.5-inch (SHIMMER GOLD) & vibrate & Sony Ericsson W995a Walkman (Progressive Black) \\
\hline sound & SKY Devices Platinum Series 5.0W - Silver & recording & ZTE Axon Pro, 64 GB Phthalo Blue \\
\hline sd & Nokia N9 16 GB MeeGo OS - Black & zoom & Nokia N9 16 GB MeeGo OS - Black \\
\hline network & ASUS ZenFone 3 ZE552KL 5.5-inch (SHIMMER GOLD) & chat & BLU Life View L110X 5.7-Inch (Blue) \\
\hline button & ASUS ZenFone 3 ZE552KL 5.5-inch (SHIMMER GOLD) & ringtone & Samsung Evergreen A667 - Black \\
\hline music & Nokia N9 16 GB MeeGo OS - Black & heats & THL 5000 5" FHD IPS MTK6592T (Black) \\
\hline internet & Nokia N9 16 GB MeeGo OS - Black & voicemail & ZTE Axon Pro, 64 GB Phthalo Blue \\
\hline light & JUNING 7-Inch - Black & stereo & BLU Life View L110X 5.7-Inch (Blue) \\
\hline warranty & Samsung Galaxy Note 5, White 32GB (AT\&T) & scroll & LG V10 H962 64GB Ocean Blue, Dual Sim, 5.7” \\
\hline bluetooth & LG VX8500 Chocolate Phone (Verizon Wireless) & guarantee & Smart watch, GEEKERA Bluetooth Watch Phone ( Black) \\
\hline video & JUNING 7-Inch - Black & languages & Sony Ericsson W995a Walkman (Progressive Black) \\
\hline settings & Asus ZenFone 3 ZE552KL 64GB Sapphire Black, 5.5-inch & multimedia & LG V10 H901 64GB T-Mobile- Space Black \\
\hline color & Nokia N9 16 GB MeeGo OS - Black & compatibility & LG Electronics G3 Stylus D690 (Black Titanium) \\
\hline design & Nokia N9 16 GB MeeGo OS - Black & driver & BLU Life View L110X 5.7-Inch (Blue) \\
\hline download & ASUS ZenFone 3 ZE552KL 5.5-inch (SHIMMER GOLD) & pedometer & Sony Ericsson W995a Walkman (Progressive Black) \\
\hline usb & SKY Devices Platinum Series 5.0W - Silver & trackpad & BlackBerry Passport (SQW100-1)Black \\
\hline email & $5.5 \%$ JUNING Blue & calculator & 5.5” JUNING Blue \\
\hline speed & Nokia N9 16 GB MeeGo OS - Black & handsfree & LG G4 5.5-Inch (Black Leather) \\
\hline headphones & ASUS ZenFone 3 ZE552KL 5.5-inch (SHIMMER GOLD) & autofocus & BLU ENERGY X - Gold \\
\hline gps & Sony Ericsson W995a Walkman (Progressive Black) & $\operatorname{otg}$ & BLU ENERGY X - Gold \\
\hline games & Nokia N9 16 GB MeeGo OS - Black & troubleshooting & Sony Xperia sola MT27i-BLK (Black) \\
\hline ram & ASUS Zenfone 6 A600CG White & airplane & Kyocera Hydro C5170 \\
\hline messages & Blackberry Torch 9800 - Black & mute & HTC One M7 - Black \\
\hline cable & Nokia N9 16 GB MeeGo OS - Black & syncs & Nokia Lumia 925 RM-893 - Black/Dark Grey \\
\hline manual & ASUS ZenFone 3 ZE552KL 5.5-inch (SHIMMER GOLD) & multitask & ZTE Axon Pro, 64 GB Phthalo Blue \\
\hline processor & ASUS ZenFone 3 ZE552KL 5.5-inch (SHIMMER GOLD) & backlight & ZTE Axon Pro, 64 GB Phthalo Blue \\
\hline specs & ASUS ZenFone 3 ZE552KL 5.5-inch (SHIMMER GOLD) & permissions & POSH Titan HD E500a - 5.0" HD, (Yellow) \\
\hline hardware & ASUS ZenFone 3 ZE552KL 5.5-inch (SHIMMER GOLD) & reminders & BLU Life View L110X 5.7-Inch (Blue) \\
\hline fingerprint & LG V10 H962 64GB Ocean Blue, Dual Sim, 5.7” & echo & Motorola Moto E (1st Generation) - Black - 4 GB \\
\hline switch & Nokia N9 16 GB MeeGo OS - Black & trackball & Blackberry Gemini 8520 White \\
\hline accessories & ZTE Axon Pro, 64 GB Phthalo Blue & panorama & ZTE Axon Pro, 64 GB Phthalo Blue \\
\hline weight & $5.5 "$ JUNING Blue & speech & Verizon LG Ally VS740 \\
\hline sensor & Nokia N9 16 GB MeeGo OS - Black & lockscreen & LG G3 D855 Black \\
\hline face & LG V10 H962 64GB Ocean Blue, Dual Sim, 5.7" & vga & BLU Win Jr Windows White \\
\hline
\end{tabular}

i.e., average, across our features. Note that not all the phones will have reviews mentioning every single feature we have selected. For example, the vga feature (at the end of the Table) is mentioned in the reviews of only 14 phones. So, only 14 phones can have ratings for the vga feature. In Table V, we rank all our phones based on the number of features in which they are the best based on the reviews. We report only those phones which are best for at least ten features. Based on these rankings, we also report the best phone for which the required feature has been rated in Table VII so that we can recommend a phone for a given feature. For example, both Nokia N9 -
Black and JUNING 7-Inch - Black have ratings of 5 for music, and we recommend Nokia N9 - Black since its best in more number of features. So, the higher the ranking, the better is the chance for the recommendation.

\section{Evaluation}

Although there are no ground-truth feature-level ratings available to evaluate our method, we have the purposely selected phone features that can be evaluated. We can compare our results on the phone named feature with the phones overall ratings already available in the dataset. The ratings given by 
TABLE VIII

DIFFERENT ERROR METRIC VALUES WHILE COMPARING THE PREDICTED RATINGS OF OUR 'PHONE' FEATURE WITH THE ACTUAL RATINGS GIVEN BY THE CUSTOMERS

\begin{tabular}{|l||l|l|l|}
\hline Error Metrics & MSE & RMSE & MAE \\
\hline Values & 0.545 & 0.738 & 0.555 \\
\hline
\end{tabular}

TABLE IX

CONFUSION MATRIX WHILE COMPARING THE PREDICTED RATINGS FOR OUR 'PHONE' FEATURE WITH THE ACTUAL INTEGER RATINGS OF PHONES

\begin{tabular}{|l|l|l|l|l|l|l|}
\hline & \multicolumn{7}{|c|}{ Predicted } \\
\hline \multirow{4}{*}{ Actual } & & 1-star & 2 -star & 3-star & 4-star & 5-star \\
\cline { 2 - 7 } & 1-star & 6 & 23 & 73 & 11 & 3 \\
\cline { 2 - 7 } & 2-star & 2 & 23 & 274 & 59 & 3 \\
\cline { 2 - 7 } & 3-star & 2 & 30 & 841 & 376 & 30 \\
\cline { 2 - 7 } & 4-star & 2 & 9 & 649 & 1184 & 72 \\
\cline { 2 - 7 } & 5-star & 0 & 3 & 60 & 295 & 111 \\
\hline
\end{tabular}

individual customers are weighted averaged (weighted by the review votes) and considered as ground-truth ratings for our phone named feature. We report different error metric values in the Table VIII while comparing with such ground truth ratings. It is impressive that, on average, our rating differs from the actual ratings by just 0.555 , which is approximately just half a star, as suggested by the MAE (Mean Average Error) error metric.

Also, we report a confusion matrix for our phone feature in Table IX Both our and ground-truth ratings are rounded to get such integer star ratings. So, we now have five classes (1-star to 5 -star) into which a phone can be classified. With such discrete outputs now, we generate the confusion matrix. It suggests that our system predicts correct ratings for 2165 mobiles and the ratings within 1-star closeness for 3886 mobiles out of a total of 4141 mobiles. So, if we want the exact integer star rating, the accuracy of the proposed method is $52.3 \%$. However, if we can tolerate the error of 1-star in the integer star rating, then accuracy jumps to $93.8 \%$. Therefore, we can comfortably say that the proposed methodology does work well for the phone named feature. Note that the total number of mobiles here has changed from 4418 . That is because there might be some phones which do not have any review with the feature words we have chosen as the words related to the phone named feature. Therefore, such phones do not receive the rating for their phone named feature to participate in this kind of evaluation, which requires at least one such review for consideration.

\section{CONCLUSION}

We have developed a system to rate mobile phones in terms of 108 features based on customer reviews and review votes. We could rate $4 \mathrm{k}+$ phones; this can help make personalized buying decisions and improve the products. We accomplish this by first converting the unstructured data into structured data; then, we extract the sentences comprising our feature keywords; then, we were able to provide the feature-level ratings through sentiment analysis of these sentences. We rank the phones based on the number of features they are best at, and accordingly, we were able to recommend the best phones for a feature. We tested our methodology on the phone named feature by considering the overall customer ratings as groundtruth ratings. The performance of our method is found to be decent. We obtain MAE of only 0.555 , i.e., approximately just half a star. We get 52.3\% accuracy if exact integer ratings have to be predicted. However, if we can tolerate the 1-star integer rating error, the accuracy jumps to $93.8 \%$. The proposed approach is unsupervised. As an extension, we will work on improving the performance by taking a weakly-supervised or supervised approach to this problem, for which we will have to annotate the available data in terms of all our 108 features.

\section{REFERENCES}

[1] V. Raghavan, G. Ver Steeg, A. Galstyan, and A. G. Tartakovsky, "Modeling temporal activity patterns in dynamic social networks," IEEE Transactions on Computational Social Systems, vol. 1, no. 1, pp. 89$107,2014$.

[2] A. Farasat, G. Gross, R. Nagi, and A. G. Nikolaev, "Social network analysis with data fusion," IEEE Transactions on Computational Social Systems, vol. 3, no. 2, pp. 88-99, 2016.

[3] Y. Zhu, D. Li, R. Yan, W. Wu, and Y. Bi, "Maximizing the influence and profit in social networks," IEEE Transactions on Computational Social Systems, vol. 4, no. 3, pp. 54-64, 2017.

[4] X. Yang, C. Liang, M. Zhao, H. Wang, H. Ding, Y. Liu, Y. Li, and J. Zhang, "Collaborative filtering-based recommendation of online social voting," IEEE Transactions on Computational Social Systems, vol. 4, no. 1, pp. 1-13, 2017.

[5] F. S. N. Karan and S. Chakraborty, "Dynamics of a repulsive voter model," IEEE Transactions on Computational Social Systems, vol. 3, no. 1, pp. 13-22, 2016.

[6] F. Smarandache, M. Colhon, Ş. Vlăduţescu, and X. Negrea, "Word-level neutrosophic sentiment similarity," Applied Soft Computing, vol. 80, pp. 167-176, 2019.

[7] K. Ravi, V. Ravi, and P. S. R. K. Prasad, "Fuzzy formal concept analysis based opinion mining for crm in financial services," Applied Soft Computing, vol. 60, pp. 786-807, 2017.

[8] J. Xu, F. Huang, X. Zhang, S. Wang, C. Li, Z. Li, and Y. He, "Sentiment analysis of social images via hierarchical deep fusion of content and links," Applied Soft Computing, vol. 80, pp. 387-399, 2019.

[9] F. H. Khan, U. Qamar, and S. Bashir, "Sentimi: Introducing point-wise mutual information with sentiwordnet to improve sentiment polarity detection," Applied Soft Computing, vol. 39, pp. 140-153, 2016.

[10] K. R. Jerripothula, J. Cai, and J. Yuan, "Quality-guided fusion-based co-saliency estimation for image co-segmentation and colocalization," IEEE Transactions on Multimedia, vol. 20, no. 9, pp. 2466-2477, 2018.

[11] A. Wiliam, W. K. Sasmoko, and Y. Indrianti, "Sentiment analysis of social media engagement to purchasing intention," in Understanding Digital Industry: Proceedings of the Conference on Managing Digital Industry, Technology and Entrepreneurship (CoMDITE 2019), July 1011, 2019, Bandung, Indonesia. Routledge, 2020, p. 362.

[12] L. G. Singh, A. Anil, and S. R. Singh, "She: Sentiment hashtag embedding through multitask learning," IEEE Transactions on Computational Social Systems, 2020.

[13] K.-P. Lin, Y.-W. Chang, C.-Y. Shen, and M.-C. Lin, "Leveraging online word of mouth for personalized app recommendation," IEEE Transactions on Computational Social Systems, vol. 5, no. 4, pp. 10611070, 2018.

[14] N. Bui, J. Yen, and V. Honavar, "Temporal causality analysis of sentiment change in a cancer survivor network," IEEE transactions on computational social systems, vol. 3, no. 2, pp. 75-87, 2016.

[15] R.-C. Chen et al., "User rating classification via deep belief network learning and sentiment analysis," IEEE Transactions on Computational Social Systems, 2019.

[16] S. Kumar, K. De, and P. P. Roy, "Movie recommendation system using sentiment analysis from microblogging data," IEEE Transactions on Computational Social Systems, 2020.

[17] M. Ling, Q. Chen, Q. Sun, and Y. Jia, "Hybrid neural network for sina weibo sentiment analysis," IEEE Transactions on Computational Social Systems, 2020.

[18] K. Chakraborty, S. Bhattacharyya, and R. Bag, "A survey of sentiment analysis from social media data," IEEE Transactions on Computational Social Systems, vol. 7, no. 2, pp. 450-464, 2020. 
[19] F.-C. Yang, A. J. Lee, and S.-C. Kuo, "Mining health social media with sentiment analysis," Journal of medical systems, vol. 40, no. 11, p. 236, 2016.

[20] M. Palomino, T. Taylor, A. Göker, J. Isaacs, and S. Warber, "The online dissemination of nature-health concepts: Lessons from sentiment analysis of social media relating to nature-deficit disorder," International journal of environmental research and public health, vol. 13, no. 1, p. $142,2016$.

[21] M. T. Khan and S. Khalid, "Sentiment analysis for health care," in Big Data: Concepts, Methodologies, Tools, and Applications. IGI Global, 2016, pp. 676-689.

[22] J. Ramteke, S. Shah, D. Godhia, and A. Shaikh, "Election result prediction using twitter sentiment analysis," in 2016 international conference on inventive computation technologies (ICICT), vol. 1. IEEE, 2016, pp. 1-5.

[23] S.-O. Proksch, W. Lowe, J. Wäckerle, and S. Soroka, "Multilingual sentiment analysis: A new approach to measuring conflict in legislative speeches," Legislative Studies Quarterly, vol. 44, no. 1, pp. 97-131, 2019.

[24] Y. Yu and X. Wang, "World cup 2014 in the twitter world: A big data analysis of sentiments in us sports fans tweets," Computers in Human Behavior, vol. 48, pp. 392-400, 2015.

[25] G. M. Lucas, J. Gratch, N. Malandrakis, E. Szablowski, E. Fessler, and J. Nichols, "Goaalll!: Using sentiment in the world cup to explore theories of emotion," Image and Vision Computing, vol. 65, pp. 58-65, 2017.

[26] S. L. Addepalli, S. G. Addepalli, M. Kherajani, H. Jeshnani, and S. Khedkar, "A proposed framework for measuring customer satisfaction and product recommendation for ecommerce," International Journal of Computer Applications, vol. 138, no. 3, pp. 30-35, 2016.

[27] J. Mehta, J. Patil, R. Patil, M. Somani, and S. Varma, "Sentiment analysis on product reviews using hadoop," International Journal of Computer Applications, vol. 9, no. 11, pp. 0975-8887, 2016.

[28] X. Fang and J. Zhan, "Sentiment analysis using product review data," Journal of Big Data, vol. 2, no. 1, p. 5, Jun 2015. [Online]. Available: https://doi.org/10.1186/s40537-015-0015-2

[29] D. K. Raja and S. Pushpa, "Feature level review table generation for ecommerce websites to produce qualitative rating of the products," Future Computing and Informatics Journal, vol. 2, no. 2, pp. 118-124, 2017.

[30] N. Nandal, R. Tanwar, and J. Pruthi, "Machine learning based aspect level sentiment analysis for amazon products," Spatial Information Research, pp. 1-7, 2020.

[31] J. Sadhasivam and R. B. Kalivaradhan, "Sentiment analysis of amazon products using ensemble machine learning algorithm," International Journal of Mathematical, Engineering and Management Sciences, vol. 4, no. 2, pp. 508-520, 2019.

[32] K. R. Jerripothula, J. Cai, and J. Yuan, "Efficient video object colocalization with co-saliency activated tracklets," IEEE Transactions on Circuits and Systems for Video Technology, vol. 29, no. 3, pp. 744-755, 2019.

[33] K. R. Jerripothula, J. Cai, J. Lu, and J. Yuan, "Object co-skeletonization with co-segmentation," in 2017 IEEE Conference on Computer Vision and Pattern Recognition (CVPR), 2017, pp. 3881-3889.

[34] K. R. Jerripothula, J. Cai, and J. Yuan, "Cats: Co-saliency activated tracklet selection for video co-localization," in Computer Vision - ECCV 2016, B. Leibe, J. Matas, N. Sebe, and M. Welling, Eds. Cham: Springer International Publishing, 2016, pp. 187-202.

[35] K. R. Jerripothula, J. Cai, F. Meng, and J. Yuan, "Automatic image co-segmentation using geometric mean saliency," in 2014 IEEE International Conference on Image Processing (ICIP), 2014, pp. 3277-3281.

[36] K. Zhang, R. Narayanan, and A. Choudhary, "Voice of the customers: mining online customer reviews for product feature-based ranking," 06 2010, pp. 11-11.

[37] K. Bafna and D. Toshniwal, "Feature based summarization of customers reviews of online products," Procedia Computer Science, vol. 22, pp. 142-151, 2013.

[38] K. R. Jerripothula, J. Cai, and J. Yuan, "Group saliency propagation for large scale and quick image co-segmentation," in 2015 IEEE International Conference on Image Processing (ICIP), 2015, pp. 4639-4643.

[39] C. J. Hutto and E. Gilbert, "Vader: A parsimonious rule-based model for sentiment analysis of social media text," in Eighth international AAAI conference on weblogs and social media, 2014.

[40] K. R. Jerripothula, J. Cai, and J. Yuan, "Qcce: Quality constrained co-saliency estimation for common object detection," in 2015 Visual Communications and Image Processing (VCIP), 2015, pp. 1-4.
[41] M. Glenski, C. Pennycuff, and T. Weninger, "Consumers and curators: Browsing and voting patterns on reddit," IEEE Transactions on Computational Social Systems, vol. 4, no. 4, pp. 196-206, 2017.

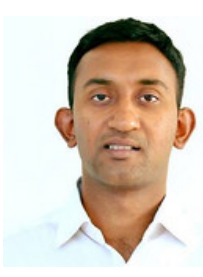

Koteswar Rao Jerripothula (Member, IEEE)) received the B.Tech. degree from Indian Institute of Technology Roorkee (IIT Roorkee), India, in 2012, and the Ph.D. degree from Nanyang Technological University (NTU), Singapore, in 2017. He is currently an assistant professor with the CSE department, Indraprastha Institute of Information Technology Delhi (IIIT Delhi), India. He has also worked with Graphic Era, ADSC (Singapore), and Lenskart earlier. His major research interests include computer vision, machine learning, and natural language processing. He was the recipient of the Top 10\% paper award at ICIP14. He has published in several top venues like CVPR, ECCV, TMM, and TCSVT.

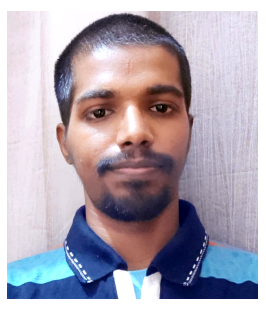

Ankit Rai received his B.Tech degree from Graphic Era, India, in 2020 . He is currently working with Infosys, India. His research interests include machine learning and natural language processing.

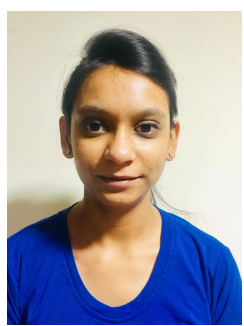

Kanu Garg received her B.Tech degree from Graphic Era, India, in 2020. She is currently working with Infosys, India. Her research interests include computer vision, machine learning and natural language processing.

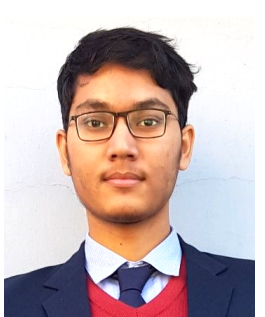

Yashvardhan Singh Rautela received his B.Tech degree from Graphic Era, India, in 2020. He is currently working with Cognizant, India. His research interests include machine learning and natural language processing. 